\title{
LANDSAT 9 THERMAL INFRARED SENSOR 2 ARCHITECTURE AND DESIGN
}

\author{
Jason H. Hair ${ }^{1}$, Dennis C. Reuter ${ }^{1}$, Synthia L. Tonn ${ }^{1}$, Joel McCorkel ${ }^{1}$, Amy A, Simon ${ }^{1}$, Melody Djam², David \\ Alexander $^{3}$, Kevin Ballou ${ }^{1}$, Richard Barclay ${ }^{1}$, Phillip Coulter ${ }^{1}$, Michael Edick ${ }^{4}$, Boryana Efremova ${ }^{5}$, Paul \\ Finneran ${ }^{6}$, Jose Florez ${ }^{4}$, Steven Graham ${ }^{1}$, Kenneth Harbert ${ }^{4}$, Dennis Hewitt ${ }^{2}$, Michael Hickey ${ }^{1}$, Samantha Hicks ${ }^{1}$, \\ William Hoge ${ }^{4}$, Murzy Jhabvala ${ }^{1}$, Carol Lilly ${ }^{7}$, Allen Lunsford ${ }^{8}$, Laurie Mann ${ }^{1}$, Candace Masters ${ }^{9}$, Matthew \\ Montanaro $^{10}$, Theodore Muench ${ }^{1}$, Veronica Otero ${ }^{1}$, Fil Parong ${ }^{1}$, Aaron Pearlman ${ }^{5}$, Jonathan Penn ${ }^{11}$, \\ Danielle Vigneau ${ }^{1}$, Brian Wenny ${ }^{12}$
}

\begin{abstract}
${ }^{1}$ NASA Goddard Space Flight Center, ${ }^{2}$ Bay Engineering Innovations, Inc, ${ }^{3}$ ASRC Federal Space and Defense, ${ }^{4}$ Florez Engineering, LLC, ${ }^{5}$ GeoThinkTank LLC, ${ }^{6}$ Jackson and Tull, Inc., ${ }^{7}$ Alcyon Technical Services, LLC, ${ }^{8}$ Catholic University of America, ${ }^{9}$ General Dynamics C4 Systems, Inc., ${ }^{10}$ Rochester Institute of Technology,

${ }^{11}$ Stinger Ghaffarian Technologies, Inc., ${ }^{12}$ Science Systems and Applications, Inc.
\end{abstract}

\begin{abstract}
The Thermal Infrared Sensor 2 (TIRS-2) will fly aboard the Landsat 9 spacecraft and leverages the Thermal Infrared Sensor (TIRS) design currently flying on Landsat 8. TIRS-2 will provide similar science data as TIRS, but is not a buildto-print rebuild due to changes in requirements and improvements in absolute accuracy. The heritage TIRS design has been modified to reduce the influence of stray light and to add redundancy for higher reliability over a longer mission life. The TIRS-2 development context differs from the TIRS scenario, adding to the changes. The TIRS-2 team has also learned some lessons along the way.
\end{abstract}

Index Terms - TIRS-2, Landsat 9, Thermal, Infrared

\section{INTRODUCTION}

Landsat 9, with its two instruments, the Operational Land Imager - 2 (OLI-2) and TIRS-2, will continue the data record of the Landsat program which dates back to 1972. TIRS-2 provides thermal band data continuity with previous Landsat thermal sensors [1]. TIRS-2 has the same measurement performance requirements as the Thermal Infrared Sensor (TIRS) flying on Landsat 8, producing radiometrically calibrated, geo-located thermal infrared image data for Landsat bands 10 and 11 centered at $10.8 \mu \mathrm{m}$ and $12 \mu \mathrm{m}$. The TIRS data is used for a number of applications including (see [1] and references therein, [2]):

- Monitoring of evapotranspiration, water use on a regional and field-by-field basis in the U.S. and internationally

- Cloud detection, screening to process OLI-2 image data

- Forestry and land use management

- Mapping urban heat fluxes for air quality modeling

- Wildfire, volcano risk assessment, mapping, recovery

- Identifying mosquito breeding areas
TIRS-2 is being developed in-house by NASA's Goddard Space Flight Center (GSFC). Landsat 9 heavily leverages the design of Landsat 8 to accelerate launch to December 2020 and for cost efficiency. Likewise, TIRS-2 uses the TIRS design to the greatest extent possible. The heart of the TIRS-2 design remains the Quantum Well Infrared Photodetector (QWIP) arrays that are cooled to below 43 kelvin by a Cryocooler Thermal Mechanical Unit (TMU). Large radiators dissipate the heat from the TMU and cool the telescope to minimize measurement background noise. The Scene Select Mechanism (SSM) keeps the instrument pointed at nadir for normal science, and rotates to deep space and an on-board black body for calibration. The TMU is controlled by the Cryocooler Control Electronics (CCE). Focal Plane Electronics (FPE) read the detector signal and sends it to the Main Electronics Box (MEB). The MEB controls the overall system in conjunction with the Mechanism Control Electronics (MCE), Command and Data Handling (CDH), High Speed Interface (HSIB), Power Control (PCB), and Thermal Control (TCB) Boards. An overview of the TIRS-2 instrument architecture is shown in Figure 1 (see [1] for more details of the TIRS design).

Of the potential TIRS design changes, only changes specifically approved through a trade study process were allowed. The sources of potential changes were changes in requirements, improvements over TIRS on-orbit performance, and an examination of TIRS waivers, problems during development, risks, and lessons learned.

TIRS-2 will be characterized extensively at the component, sub-assembly, and system level to ensure that the design meets performance requirements to achieve the science objectives $[3,4,5]$.

This paper provides an overview of the TIRS-2 development strategy and context, requirement and design changes, and lessons learned. 


\section{TIRS-2 DEVLEOPMENT CONTEXT \& STRATEGY}

NASA gave the Landsat 9 Project, as well as the TIRS-2 Instrument Project, authority to begin work in March 2015 with direction to achieve the same performance requirements as Landsat 8, develop TIRS-2 as a Class B instrument, capitalize on Landsat 8 design, engineering, and hardware assets, complete the development with a cost substantially less than Landsat 8, and launch by 2020.

Consistent with this direction, the TIRS-2 team formulated a project plan to meet the schedule challenge, maintain a low cost, and achieve the performance requirements. The overall strategy was to minimize changes from TIRS in order to contain schedule, cost, and risk. The team allowed only the changes that were necessary and specifically approved. While there was much focus on minimizing design changes, the team put equal emphasis on maintaining interfaces and maximizing the use of TIRS processes, procedures, spare hardware, test plans, and Ground Support Equipment (GSE). The TIRS-2 team recognized early that key parts of the design, such as the telescope, filters, detectors, and cryocooler, had to remain substantially the same since they formed the heart of the instrument. Any changes to one would have trickle-down impacts to many other areas. For example, the telescope lenses could not be changed to reduce the ground sample size as this would require more detectors and increased cooling capacity from the cryocooler and radiators.

Although TIRS-2 leverages the TIRS design, it is not a build-to-print rebuild due to top-level requirement changes and differences in the paradigm for development of TIRS and TIRS-2. TIRS was designed as for Class $\mathrm{C}$ reliability with a 3.25 year mission life. TIRS completed design, build, and test on an accelerated schedule (3 years) to meet the planned Landsat 8 launch date. Working in that scenario, the TIRS team had to take risks and did not have the luxury of running every question to a solution in order to meet the tight schedulel. With this in mind, the TIRS-2 team put significant effort into reviewing TIRS as-built documentation, lessons learned, deviations, waivers, nonconformances, residual risk, and on-orbit performance as sources of changes for TIRS-2, in addition to the requirements-driven changes. As part of this process, a lessons learned workshop was held with TIRS and TIRS-2 staff for open dialog at the subsystem level. Trade studies were used to vet and approve design and process changes. A Heritage Review (HR) was held early to review requirements changes; disposition of lessons learned, waivers, non-conformances, and residual risk; trade study results; parts of the design that would remain unchanged; design and process changes; and the key TIRS hardware and GSE that would be re-used by TIRS-2. After the HR, TIRS2 followed a more traditional review process with a Preliminary Design Review (PDR) and a Critical Design Review (CDR). Detailed Engineering Peer Reviews at the subsystem level were held prior to PDR and CDR to facilitate a detailed review of designs, analyses, and plans.

Where possible, TIRS-2 used residual TIRS hardware and documentation after a review and approval process. For any inherited TIRS hardware item proposed to be used as a fight or flight spare unit for TIRS-2, the documentation, pedigree, prior test history, and any non-conformances were captured, reviewed, and approved by cognizant engineering, mission assurance, and reliability personnel. TIRS-2 will fly with TIRS flight spare hardware in some cases, such as QWIP Sensor Chip Arrays (SCAs) that were assembled into new Focal Plane Array (FPA), filters, SSM motors, SSM bearings, and many electronic parts. TIRS flight spare hardware was also maintained as TIRS-2 flight spares while new hardware for TIRS-2 was built. TIRS GSE is being reused extensively, including MEB card electronic racks, calibration test equipment (CalGSE), and assembly tooling and fixtures. The TIRS document control system was copied and linked to the TIRS-2 document control system to facilitate reuse. For TIRS parts to be remade with no changes, the drawings were approved by TIRS-2 personnel and released in the TIRS-2 system via an expedited process. A new TIRS-2 drawing number was issued for new or modified parts. TIRS-2 work instructions can be initiated by cloning a TIRS work instruction, then updated, reviewed, and approved by the TIRS-2 team.

As the TIRS- 2 team was assembled, about $40 \%$ of the key personnel also worked on TIRS, which, along with consulting support by other TIRS personnel, greatly aided in the continuity of knowledge and facilitated a faster learning curve for the rest of the TIRS-2 staff. Another key aspect to the project's success is that the entire TIRS-2 team collaborated to raise the team's collective level of understanding of the TIRS design to match that of the original TIRS design team.

\section{TIRS-2 REQUIREMENTS AND DESIGN CHANGES}

The major requirements changes for TIRS-2 are:

- Increase the reliability requirement to Class B with a 5.25 year mission life (from Class C, 3.25 year mission life)

- Increase in launch design limit environments

- Improve survival temperature limit

- Improve micrometeorite protection

- Increase surface charge dissipation

To achieve the new reliability requirement, redundancy and cross-strapping were added to the electrical system architecture and changes were made to the thermal control system, as shown in Figure 1. TIRS-2 consists of two MEBs and two CCEs, plus existing two-sided FPE, to make for full redundancy. The single cryocooler TMU is connected via the new Redundancy Switch Electronics (RSE) to the two CCEs, which are cross-strapped to the two MEBs. The MEB 
is cross-strapped to the FPEs via the new FPE Interface Box (FIB) and to the SSM encoder. Either MEB can communicate with either FPE, such that a calibrated FPE can remain linked with the detector even if another part of the system has a problem and has to switch to the redundant side. On-orbit, TIRS had to change sides due to the encoder, but was forced to also change sides of the FPE due to the design, requiring an extensive recalibration effort. Temperature control zones were added to TIRS-2 to ensure fully redundant control with margin. Temperature sensors previously used only for telemetry were moved to the TCB from the $\mathrm{CDH}$ so they could also be used for control if the primary sensor failed. Due to the added heater services, the TCB had to be updated extensively to provide the required power, dissipate the heat, and allow control sensors to be selected via command. The telescope radiator area was increased and the telescope operational temperature was raised by 5 kelvin to allow for positive control with margin using only one side at a time.

Due to the uncertainty of the spacecraft and launch vehicle selections, Landsat 9 decided to increase the design limit environments above the levels that TIRS had been designed and qualified to. This caused the entire TIRS-2 system to be reanalyzed to the new environment. Based on that analysis, a number of structure and subsystem element designs had to be changed to increase their strength.

Landsat 9 directed TIRS-2 to ensure that the system would survive a thermal environment induced when the spacecraft solar array was stuck in a worst-case position and the spacecraft entered safe hold mode. To conform to this requirement, survival heaters had to be added to the TIRS-2 structure and the composite bond joints had to be redesigned on the Earth shield and structure. Detailed, 3D finite element analysis was performed on most bonded joints to validate margins at the temperature extremes.

To meet the updated micrometeorite and orbital debris and surface charging requirements, a denser material was added to select ram-facing thermal blankets, the outer layer of thermal blankets was changed to be conductive, and radiator surfaces were coated with conductive coatings.

The development of TIRS-2 has also been informed by the on-orbit performance of TIRS. TIRS requires a correction for scene-dependent stray light [6]. To minimize the stray light in the TIRS-2 design, baffles were added inside the Telescope to improve the absolute accuracy and reduce or eliminate the correction. The design of the baffles have been extensively analyzed by two independent groups, and validated through lab and flight-like performance tests [5]. To facilitate the testing, the TIRS CalGSE was modified to allow for sampling an adequate range of angles to envelope the stray light features. Also, the TIRS encoder has experienced current draw increases and its operation has been restricted to occasional calibration checks, down from fulltime active control of the scene select mirror. The TIRS-

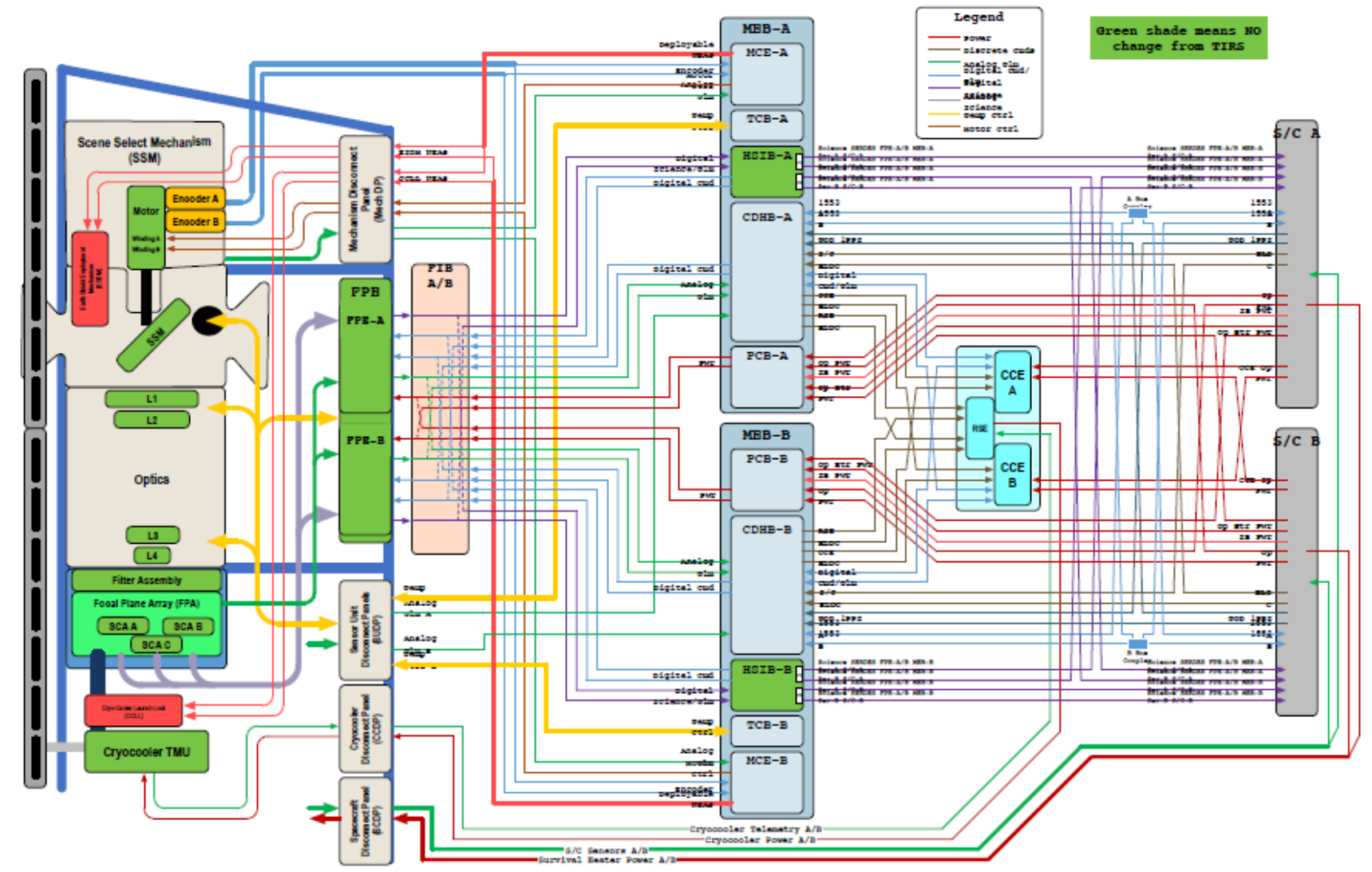

Figure 1: TIRS-2 Overall Architecture. 
2 team performed an extensive series of tests to determine the proximate cause to be current leakage in a capacitor due to a lot problem. This lot of capacitors has been expunged from inventory so they are not used on TIRS-2. Identification of this problem has also informed risk decisions on a number of other GSFC missions. When the encoder vendor had to be selected, the proximate cause was not yet known, so a new vendor was selected, increasing TIRS-2 development risk. This vendor has successfully qualified the encoder and delivered the flight unit, which has been integrated into the system.

The evaluation of TIRS lessons learned, deviations, waivers, non-conformances, as-built documentation, and residual risks contributed to additional TIRS-2 design changes. The most notable is that the detector to telescope mount was updated to be kinematic to improve repeatability to avoid stability issues that added tests during the focus setting process on TIRS. The CCE design was improved over the TIRS design based on internal vendor research and development, which contributed to CCE development success for TIRS-2. The Cryocooler control algorithms were updated to remove position sensors, eliminating a single point failure mode [7]. A new TMU fill tube plug and seal design was qualified and implemented to avoid the pinch tube leak experienced on TIRS. TIRS had 40 requirement waivers. Due to small changes in requirements, design, or test, TIRS-2 anticipates only 8 deviations and 3 waivers.

\section{TIRS-2 DEVELOPMENT LESSONS LEARNED}

All projects to deliver space flight hardware are challenging and many things do not go according to plan. The toughest lesson learned so far is that even proven interfaces that have not changed as part of a follow-on build must be adequately vetted and tested as early as possible to burn down interface functionality risk, just like new interfaces. The FPA, FPE, and the harness between them had not changed from TIRS to TIRS-2 and all was expected to work without issue. An interface test of the flight FPE with spare FPA was conducted with good results. However, the first time that an FPE was used to operate the flight FPA with flight-like harness, the system did not perform. After weeks of investigating the issue and following possible causes based on 'knowns' of the system, it was discovered that one of the connections between the FPE and FPA that was 'known' to be connected and work for TIRS, was actually disconnected during TIRS development and flight. This change in configuration on TIRS was not captured in the as-built documentation, so it turned out that the TIRS-2 team had not duplicated the interface as intended.

All changes have consequences and oftentimes the consequences are difficult to predict and account for at the time the change is made. All changes require qualification, so each change increases the project risk that a problem will be found during the verification process, requiring more time and funding to rework. Always use caution when making changes and ensure that they are necessary to warrant the possible downstream impacts.

When starting a rebuild, it is important to understand that the new project is not going to be able to take the same risks that the heritage project took. This is mostly due to the fact that more risks have to become accepted near the end of a development because there are not enough resources remaining to resolve them. Differences in risk class are also a factor. Ensure that adequate resources are available to address risks and unknowns, even when there is pressure to reduce cost and schedule for a rebuild.

\section{CONCLUSION}

TIRS-2 has carefully made strategic changes to the TIRS design to respond to changes in requirements and improve science performance. The outstanding progress made so far on TIRS-2 is directly attributable to the excellent efforts of the $300+$ individuals supporting TIRS-2 across the country. The next steps are full instrument integration, system level performance test, and delivery by August 2019.

\section{REFERENCES}

[1] D.C. Reuter, C. Richardson, J. Irons, et al., "The Thermal Infrared Sensor on the Landsat Data Continuity Mission," Proceedings of IEEE Geoscience and Remote Sensing Symposium, pp. 754-757, 2010.

[2] J.M. Irons, J.L. Dwyer, J.A. Barsi, “The next Landsat satellite: The Landsat Data Continuity Mission,” Remote Sensing of Environment, 122, pp. 11-21, 2012.

[3] J. McCorkel, M. Montanaro, B. Efremova, A. Pearlman, Brian Wenny, et al., "Landsat 9 Thermal Infrared Sensor 2 Characterization Plan Overview,” These Proceedings, 2018.

[4] B. Efremova, A.J. Pearlman, J. McCorkel, M. Montanaro, M. Hickey, A. Lunsford, D.C. Reuter, "Landsat 9 Thermal Infrared Sensor 2 Preliminary Spectral Test Results,” These Proceedings, 2018.

[5] M. Montanaro, J. McCorkel, J. Tveekrem, J. Stauder, J.H. Hair, D.C. Reuter, "Landsat 9 TIRS-2 Preliminary Stray Light Assessment,” These Proceedings, 2018.

[6] A. Gerace, M. Montanaro, "Derivation and Validation of the Stray Light Correction Algorithm for the Thermal Infrared Sensor onboard Landsat 8," Remote Sensing of Environment, 191, 246-257, 2017.

[7] E. Marquardt, R. Taylor, J. Marquardt, T. Muench, "Qualification Test Results for the TIRS-2 Cryocooler," Cryocoolers, 20, 2018. 